\title{
Fluid-structure interaction in underwater acoustics
}

\author{
J T Oden, L Demkowicz, and J Bennighof
}

Texas Institute for Computational Mechanics, University of Texas at Austin, Austin TX 78712

A short review of an ongoing work on lincar acoustics problems in TICOM is presented.

\section{Introduction}

The following is a brief summary on three different research subjects currently being investigated at 'TICOM:

(a) Application of $h-p$ Adaptive Finite Element Methods to solve transient acoustic equations.

(b) Solution of the coupled linear elasticity/ boundary Helmholtz integral equation prob. lem by means of $h-p$ adaptive finite elements, and

(c) Domain decomposition methods with application to transient analysis of structure/acoustical medium models

\section{An Adaptive Approach for Solving Transient Acous- tics Problems}

As a starting point for the analysis, we accept the system of first order linear hyperbolic equations of the classical acoustics:

$$
\begin{aligned}
& \rho_{t}+\rho_{0} \operatorname{div} u=0 \text { such that } \\
& u_{t}+\frac{c_{0}^{2}}{\rho_{0}} \operatorname{grad} \rho=0
\end{aligned}
$$

where $\rho$ and $u$ are the perturbations in density and velocity of a fluid at rest with density $\rho_{0}$ and speed of sound $c_{0}$. Equivalently, in a vector form for a two-dimensional case, we have

$$
\left\{\begin{array}{l}
\text { Find } U=\left(\rho \cdot u_{1}, u_{2}\right)^{T} \text { such that } \\
U_{, t}+A U_{, x}+B U_{, y}=0
\end{array}\right.
$$

where commas denote the differentiation and $\boldsymbol{A}$ and $B$ are appropriately defined (constant) Jacobian matrices.

The system of classical acoustic equations is obtained by linearizing the isentropic compressible Euler equations around an equilibrium stage $\boldsymbol{U}_{0}=$ $\left(\rho_{0}, 0,0\right)$ and it can be easily generalized to the case of an arbitrary "background" incompressible flow vector $\boldsymbol{U}_{0}$ (see, e.g., Majda (1984)). Jacobian matrices $A$ and $B$ become then functions of the background flow vector $V_{0}$. By replacing the Euler equations with $\mathrm{N}$ avier-Stokes equations, viscous effects can be included as well.

An initial boundary value problem for system (2) is then formulated in an exterior domain $\Omega=$ $\mathbb{R}^{2} \backslash \Omega_{0}$, where $\Omega_{0}$ is the domain occupied by a vibrating body or a rigid scatterer and appropriate boundary conditions are applied both at the surface $\partial \Omega_{1}$ of the body and at infinity (Sommerfeld radiation condition). In practice, the infinite domain is truncated to a bounded. computational domain and an artificial "open" boundary is introduced. The following boundary conditions are accepted for 
practical computations:

(a) solid wall

$$
u_{n}=\hat{u}_{n} \quad \text { on } \partial \Omega_{1}
$$

(b) symmetry of the first kind

$$
\frac{\partial \rho}{\partial n}=\frac{\partial u_{r}}{\partial n}=0, \quad u_{n}=0 \quad \text { on } \partial \Omega_{2}
$$

(c) symmetry of the second kind

$$
\frac{\partial U}{\partial n}=0 \quad \text { on } \partial \Omega_{2}
$$

where $u_{n}$ and $u_{\tau}$ denote the normal and tangential components of the velocity vector on the boundary, $\partial \backslash \partial n$ is the normal derivative, and $\hat{u}_{n}$ is a given function prescribing the vibrations of the body.

The problem is completed by initial conditions of the form

$$
u(0)=\bar{u} \text { and } \rho(0)=\bar{\rho}
$$

with $\bar{u}$ and $\bar{\rho}$ given.

Starting with a second order finite difference approximation in time

$$
\begin{aligned}
& U(t+\Delta t)=\alpha \frac{\Delta t^{2}}{2} U_{, t t}(t+\Delta t)=U(t) \\
& +\Delta t U_{, t}(t)+(1-\alpha) \frac{\Delta t^{2}}{2} U_{, t t}(t)+0\left(\Delta t^{3}\right)
\end{aligned}
$$

$$
\alpha \in[0,1]
$$

and using equations (2) to represent the time derivatives in terms of the spatial derivatives, we replace the original transient equations with a sequence of elliptic-like PDE's to be solved at time levels $n \Delta t, n=1,2,3$, of the form:

$$
\begin{aligned}
& U^{n+1}-\alpha \frac{\Delta t^{2}}{2}\left[\left(A^{2} U_{, x}^{n+1}+A B U_{, x}^{n+1}\right.\right. \\
& \left.+\left(B A U_{, x}^{n+1}+B^{2} U_{, y}^{n+1}\right)_{, y}\right]=U^{n} \\
& =\Delta t\left(A U_{, x}^{n}+B U_{i y}^{n}\right) \\
& +(1-\alpha) \frac{\Delta t^{2}}{2}\left[\left(A^{2} U_{, x}^{n}+A B U_{, y}^{n}\right)_{, x}\right. \\
& \left.+\left(B \boldsymbol{A} U_{, x}^{n}+B^{2} U_{, y}^{n}\right)_{, y}\right]
\end{aligned}
$$

where $U^{n}$ is the solution at time $t=n \Delta t$. Multiplying $(7)$ by a test function $V$. integrating over $\Omega$ and incorporating boundary conditions $(3) \div(5)$ into the boundary terms results in a classical variational formulation for (7).

The variational formulation serves then as a basis for FE discretization in space variables using the $h-p$ approximations described in Demkowicz, Oden, et al. (1989). Solution of every single time step problem is done adaptively by monitoring the FE discretization error and adapting the mesh using both $h$-p refinements and unrefinements. Adaptivity is based on the element residual technique prescribed in Oden, Demkowicz, et al. (1989, 1990).

\section{A Numerical Example}

The classical example of a vibrating cylinder was used to verify the method. With

$$
\hat{u}_{n}=\mathrm{A} \sin \omega t[H(t)-H(t-T / 2)]
$$

where $A$ is given amplitude, $\omega$ - the frequency with a corresponding period $T$ and $I(t)$ denoles the Heaviside function, the exact solution takes the form of a short signal propagating axisymmetrically from the cylinder to infinity.

A section of a circular domain displayed in Fig. 1 was selected as a computational domain with the 
indicated boundary conditions.

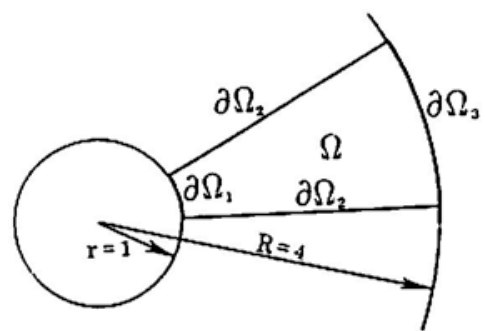

Figure 1: Vibrating cylinder problem. Selection of the computational domain and boundary conditions.

Typical results representing the density component with a corresponding FE mesh are shown in Fig 2 ( $h$-adaptivity) and in Fig. 3 (a combined $h$ $p$ adaptivity).

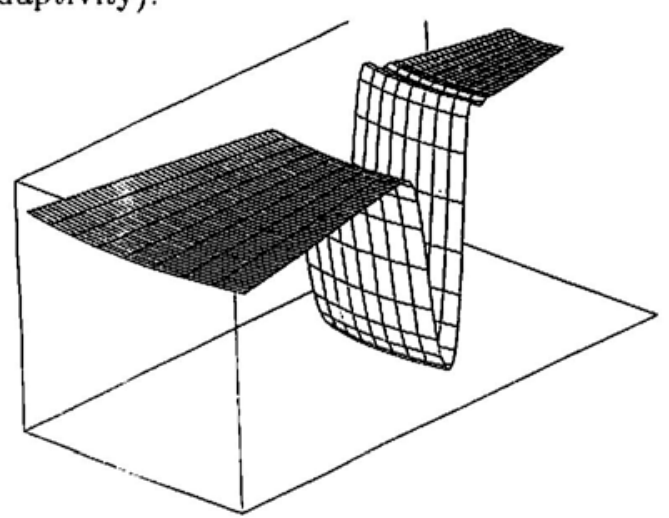

Figure 2: Vibrating cylinder problem. Pressure profile at $t=1.5$ (an h-adaptive strategy).

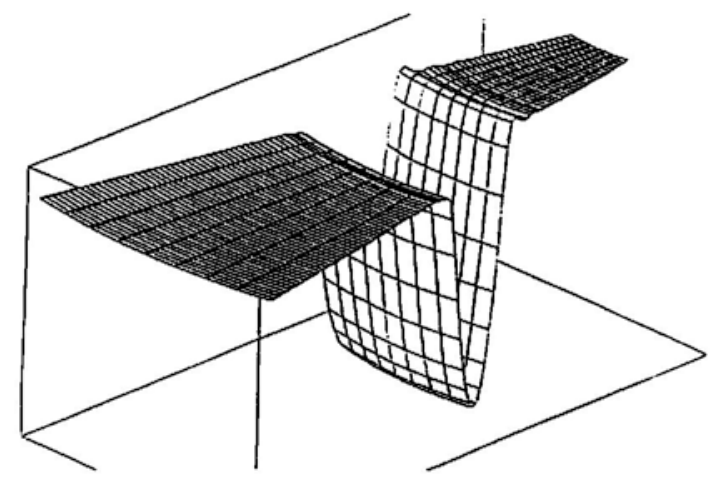

Figure 3: Vibrating cylinder problem. Pressure profile at $t=1.5$ (an $h-p$ adaptive strategy).

\section{Coupled Linear Elasticity / Boundary Helmholtz Inte- gral Equation Formulation}

A typical elastic scattering formulation for the underwater acoustics (see Fig. 4) consists of solving the linear acoustics equations in the exterior domain $\Omega^{e}$ coupled with linear elasticity equations in the interior domain $\Omega^{i}$ and accompanied by compatibility conditions on boundary $\Gamma$ and Sommerfeld radiation condition corresponding to an incident pressure and velocity field at infinity

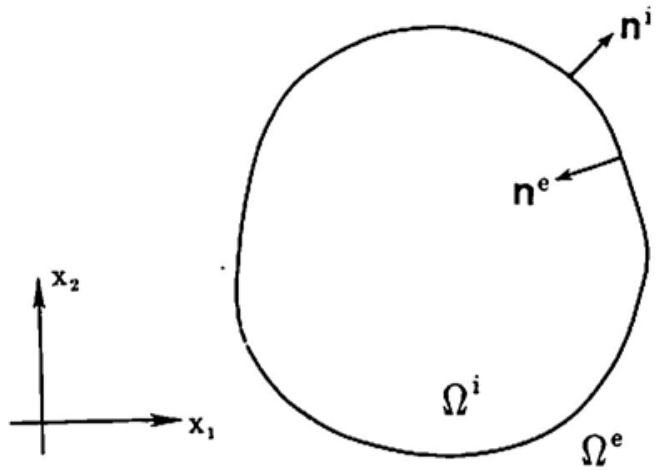

Figure 4: An elastic scattering problem. Notation

Employing the harmonic time analysis and reducing the exterior acoustics problem to the Helmholtz boundary equation, we end up with the following formulation

Find $u=u(x), x \in \Omega^{i}, p=p(x), x \in \Gamma$ such that

$-\left(E_{i j k l} u_{k, l}\right)_{, j}-\rho_{s} w^{2} u_{i}=0 \quad$ in $\Omega^{i}$

$p^{\text {inc }}+\frac{1}{2 \pi} \int_{\Gamma}\left(p \frac{\partial \Phi}{\partial n_{e}}-\Phi \frac{\partial p}{\partial n_{e}}\right) d s$

$=\frac{\sigma}{2 \pi} p$ on $\Gamma$

$$
\left.\begin{array}{l}
\sigma_{n}=-p \\
\sigma_{\tau}=0 \\
\omega^{2} u_{n}=-\frac{1}{\rho f} \frac{\partial p}{\partial n_{e}}
\end{array}\right\} \text { on } \Gamma
$$

where 


$$
\begin{array}{lll}
u=u(x) & - & \text { displacement vector } \\
p=p(x) & - & \text { acoustical pressure } \\
E_{i j k l} & - & \text { elasticities } \\
\rho_{s} & - & \text { solid density } \\
\omega & - & \text { frequency } \\
\rho_{f} & - & \text { fluid density } \\
p^{\text {inc }} & - & \text { incident pressure } \\
\sigma & - & \begin{array}{l}
\text { angle of the tangent cone on } \\
\text { the boundary (= } \pi \text { for smooth }
\end{array} \\
\frac{1}{2 \pi} \Phi & \begin{array}{l}
\text { surfaces) } \\
\frac{1}{2 \pi} \frac{\partial \Phi}{\partial n_{e}}
\end{array} & \begin{array}{l}
\text { fundamental solution to the } \\
\text { wave equation }
\end{array} \\
& \begin{array}{l}
\text { normal derivative of the } \\
\text { fundamental solution }
\end{array}
\end{array}
$$

(comp. Junger, Feit, 1986).

Replacing the elasticity equations with a "spring model," one obtains a simplified model of the form

$$
\begin{aligned}
& \sigma_{n}=-\frac{1}{\varepsilon} u_{n} \Rightarrow \frac{\partial p}{\partial n_{e}}=\varepsilon \rho_{f} \omega^{2} p \text { on } \Gamma \\
& p^{\text {inc }}+\frac{1}{2 \pi} \int_{\Gamma}\left[\frac{\partial \Phi}{\partial n_{e}} p-\Phi \frac{\partial p}{\partial n_{e}}\right] d s=\frac{\sigma}{2 \pi} p \text { on } \Gamma
\end{aligned}
$$

which, in the limiting case of spring constant $\varepsilon=0$, results in the classical Helmholtz boundary equation for rigid scattering problems:

$$
p^{\text {inc }}+\frac{1}{2 \pi} \int_{\Gamma} p \frac{\partial \Phi}{\partial n_{e}} d s=\frac{\sigma}{2 \pi} p \text { on } \Gamma
$$

Each of the problems then can be formulated in a weak form. For the 'clastic spring' model. (smooth boundary case!), for instance, we have

$$
\left\{\begin{array}{l}
\text { Find } p \text { and } \frac{\partial p}{\partial n_{e}} \text { such that } \\
\int_{\Gamma} p^{\text {inc }} \delta p+\frac{1}{2 \pi} \int_{\Gamma} \int_{\Gamma}\left[\frac{\partial \Phi}{\partial n_{e}} p-\Phi \frac{\partial p}{\partial n_{e}}\right] \delta p d s \\
=\frac{1}{2} \int_{\Gamma} p \delta p \quad \forall \delta p \\
\int_{\Gamma}\left(\frac{\partial p}{\partial n_{e}}-\varepsilon \rho_{f} \omega^{2} p\right) q=0 \quad \forall q
\end{array}\right.
$$

These variational formulations serve. in turn, as a basis for FE $h$ - $p$ approximations.

\section{A Numerical Example}

The classical problem of scattering a plane wave with a rigid cylinder shown in Fig. 5 was selected as a test case.
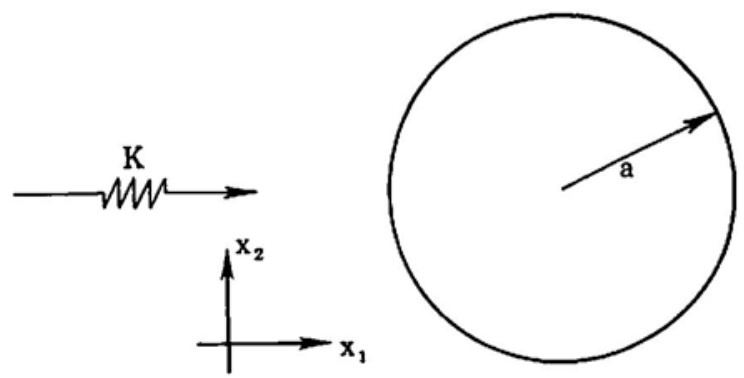

Figure 5: Scattering of a plane wave by a rigid cylinder.

Figures 6 and 7 show typical results representing the calculated total pressure on the cylinder and 
compared with the exact solution.

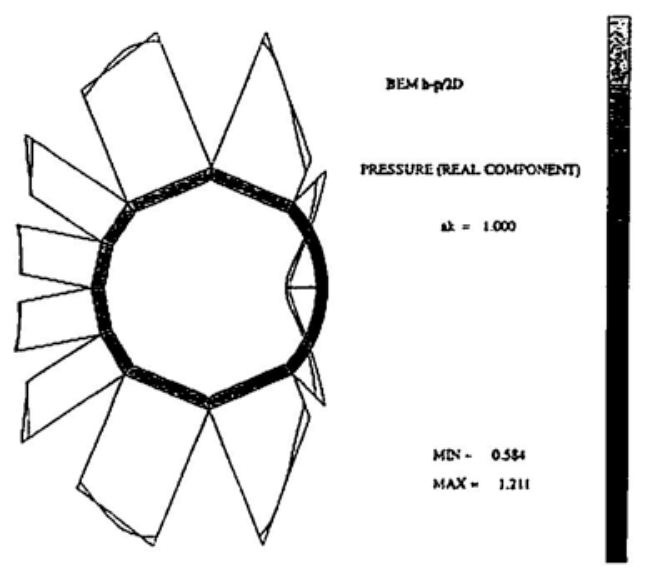

Figure 6: Scattering by a rigid cylinder. FE and exact pressures for $k a=1$ on an $h-p$ mesh.
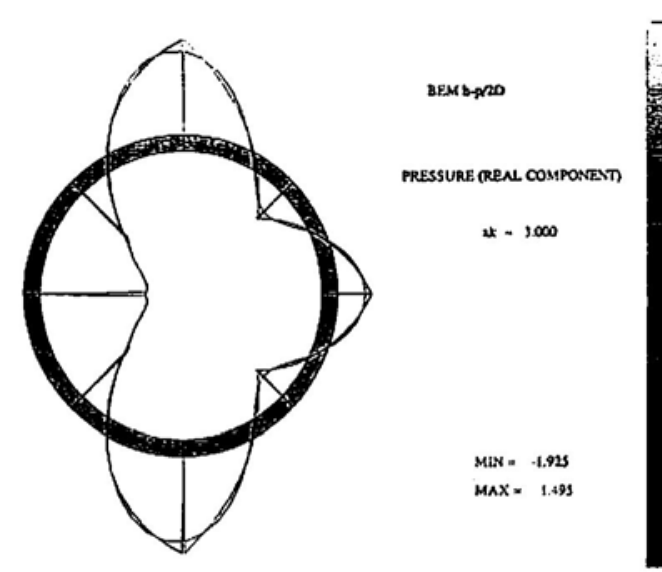

Figure 7: Scattering by a rigid cylinder. $F E$ and exact pressures for $k a=3$ on a uniform $F E$ mesh of quadratic elements.

The present effort focuses on a posteriori error estimation for the boundary element method as well as other Helmholtz integral formulations.

\section{Domain Decomposi- tion Methods for Transient Analysis}

Because of the computational requirements associated with the solution of transient problems in structural acoustics, and the impending availability of inexpensive high-performance multiprocessor computers, the potential impact of parallel computation on the solution of large transient problems is obvious. Research on parallel computation for transient problems has focused on the development of a domain decomposition algorithm in which the problem domain is partitioned into subdomains that can be assigned to different processors, and computation of transient response for subdomains can proceed independently for multiple time steps at a time $[1,2]$. At present, the algorithm has only been applied to computation of transient response of structures. Extension to the fluid part of the domain appears to be straightforward.

Space does not permit a detailed explanation of the algorithm, but it will be briefly described and illustrated with an example. 'The structure whose transient response is to be obtained is partitioned into substructures for parallel computation. The response of the structure is computed using a two step approach. First, response is computed independently for each of the substructures, neglecting the coupling between substructure responses that is present in the response of the overall structure. In the second step, the independently computed substructure responses are corrected so that they represent the response of the coupled structure model. The method for making the correction requires only a small amount of computation and communication between substructure processors. After the responses of substructures have been computed independently for, say, fifty time steps, the only information that must be communicated between processors is the interface portions of substructure responses from every tenth time step or so. This very limited amount of information can be used to determine the response of the coupled structure in a very accurate and efficient manner. The procedure for correcting the interface motion for substructures is direct, rather than iterative, and reduces to a matrix-vector multiply at the end of a time interval over which substructure responses have been com- 
puted, where a vector containing sampled substructure interface response, computed independent.ly, is multiplied by a matrix to obtain the corrected interface motion at the end of the time interval. Obtaining the corrected motion for all of the non-interface degrees of freedom in the structure also requires a similar matrix-vector multiply, where the same vector of sampled interface responses is involved. Some overhead is required for generating the matrices involved at the start of the computations.

A simple example is presented to demonstrate the performance of the algorithm. Figure 8 shows an 88-degree-of-freedom plane truss structure and its division into two substructures.

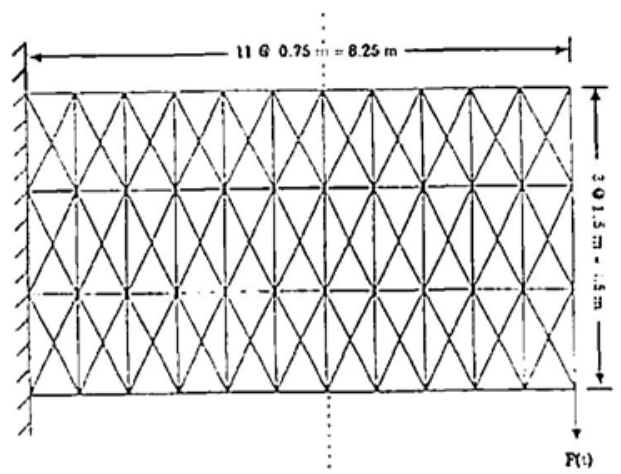

Figure 8: A plane truss problem. Division into substructures.

The truss is excited by a time-varyling force which is applied at the upper right corner and starts at zero time. Figure 9 shows the response at the point where the force is applied.

The dashed line is the exact response from a closed-form solution, and the asterisks show the results obtained using the parallel transient response algorithm. There are sixty time steps between every pair of adjacent asterisks, because this was how infrequently corrections had to be made to the responses computed independently for substructures. (lt is straightforward to obtain corrected response for any degree of freedom at any given time in the response history if desired, at some small additional computational expense.) In this example, response was computed independently for substructures for sixty time steps at a time, and the computed interface responses for substructures for every tenth time step were then used to correct the substructure responses and obtain the response for the assembled structure. The corrected structure response gives initial conditions for ongoing computation of substructure response. The accuracy obtained is quite good for the small amount of effort and communication involved in correcting the independent substructure calculations.

Ongoing research is addressing the stability of the algorithm and the speedup resulting from parallel implementation.

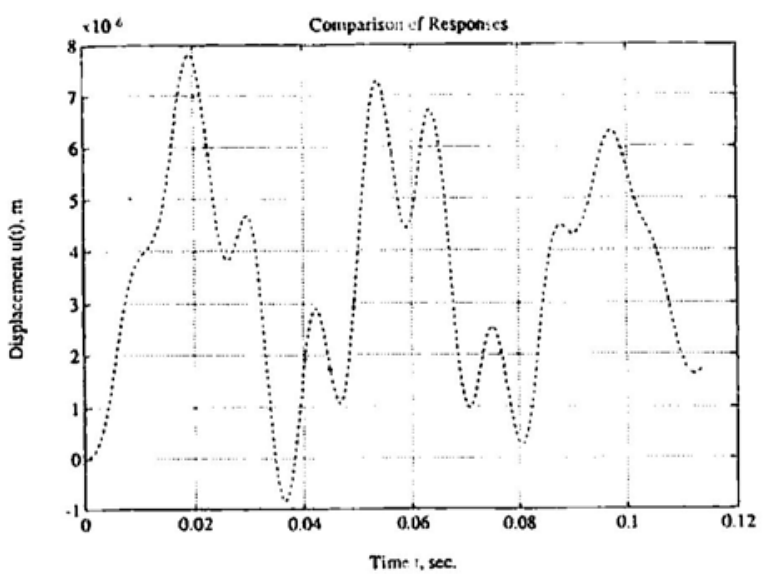

Figure 9: A plane truss problem. Comparison of responses.

\section{References}

1. Bennighof, J. K. and Wu, J. Y., "A Substructure-Based Parallel Algorithm for Structure Transient Response Problems," 30th AIAA/ASME/ASCE/AHS/ASC Structures, Structural Dynamics and Materials Conference. Mobile. Ala., April 1989, to appear, AIAA Journal. 
2. Bennighof, J. K. and Wu, J. Y., "A Parallel Structure Transient Response Algorithm Using Independent Substructure Response Computation," 3rd Annual Conference on Aerospace Computational Control, Oxnard, Calif., August 1989. To appear, Communications in Applied Numerical Methods.

3. Demkowicz, L., Oden, J. T., Rachowicz, W., and Hardy, O., "Toward a Universal $h$ $p$ Adaptive Finite Element Strategy: Part I. Constrained Approximation and Data Structure," Computer Methods in Applied Mechanics and Engineering, Vol. 77, pp. 79-112, 1989 .

4. Junger, M. C. and Feit, D., Sound, Structures and Their Interaction, the MIT Press, Cambridge, 1986.

5. Majda, A., Compressible Fluid Flow and Systems of Conservation Laws in Several Space Variables, Applied Mathmatical Sciences, Vol. 53, Springer-Verlag (New York), 1984.

6. Oden, J. T., Demkowicz, L., Rachowicz, W., and Westermann, T., "Toward a Universal $h$ p Adaptive Finite Element Strategy: Part II. A Posteriori Error Estimation," Vol. 77, pp. 113-180, 1989.

7. Oden, J. T., Demkowicz, L., Rachowicz, W., Westermann, T., "A Posteriori Error Analysis in Finite Elements: The Element Residual Method for Symmetrizable Problems with Applications to Compressible Euler and NavierStokes Equations," Computer Methods in Applied Mechanics and Engineering, to appear. 